
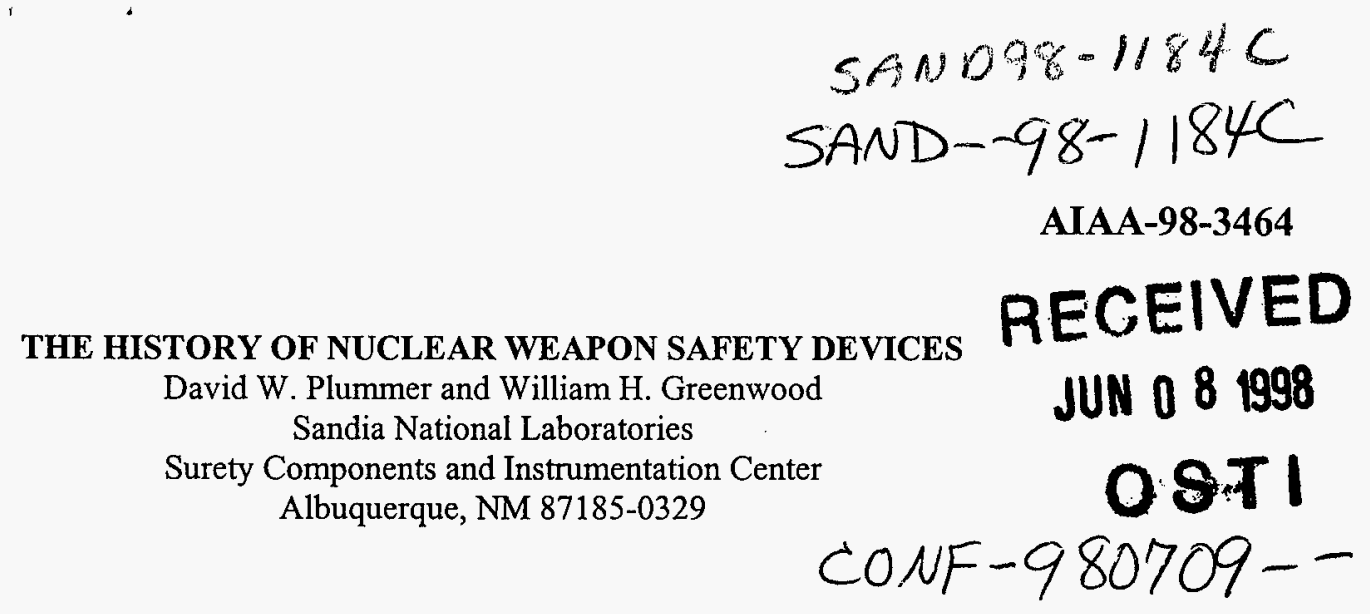

Abstract

The paper presents the history of safety devices used in nuclear weapons from the early days of separables to the latest advancements in MicroElectroMechanical Systems (MEMS). Although the paper focuses on devices, the principles of Enhanced Nuclear Detonation Safety implementation will also be presented.

\section{Original Concepts}

Nuclear weapons are composed of detonators, chemical explosives, and special nuclear material controlled by the arming, fuzing, firing, and safety systems. Nuclear weapons are packaged as bombs, ballistic missiles, and cruise missiles. With early models of nuclear weapons, it was standard procedure during most operations to keep a capsule of nuclear material separate from the weapon for safety purposes. While a weapon with the capsule removed did contain a small quantity of natural uranium with an extremely low level of radioactivity, accidental detonation of the high explosive element would not cause a nuclear detonation or significant contamination.

The philosophy of "separables" was the ultimate in nuclear detonation safety because the weapon did not physically exist until the items were assembled. Assembly was to be performed at the last moment, frequently during deployment, say in the cargo bay of an aircraft en route to the target. For example, a bomber crash near Albuquerque, NM in 1950 had a nuclear bomb aboard but there was no danger of nuclear detonation since the nuclear material was separated from the bomb body.

As tension during the cold war mounted and the risk of an unannounced first strike increased, it became impractical to assemble and deploy weapons in the time allowed. Nuclear weapons were redesigned and integrated into delivery systems to be ready immediately when needed. However, the same level of protection and assurance was required against inadvertent detonation during an accident.

\section{Motivation for Change}

Between 1950 and 1980, United States nuclear weapons were involved in 32 accidents. ${ }^{1}$ There has never been even a partial inadvertent U. S. nuclear detonation despite the very severe stresses imposed upon the weapons involved in these accidents. Only two accidents, those at Palomares, Spain, and Thule, Greenland, resulted in a widespread dispersal of nuclear materials. An "accident involving a nuclear weapon" is defined as any unexpected event that results in any of the following: accidental or unauthorized launching, firing, or use of a nuclearcapable weapon system which could create the risk of an outbreak of war; nuclear detonations; non-nuclear detonation, burning of nuclear material; radioactive contamination; seizure, theft, or loss of a nuclear weapon; or any actual or implied public hazard.

Several accidents during the 1960s focused the debate on nuclear weapon detonation safety. Accidents at Goldsboro, NC; Palomares, Spain; Thule, Greenland; and Damascus, Arkansas are the better known events. The accidents share some common themes. They all exposed the weapon to conditions that mimicked the deployment conditions and they involved extreme environmental insult, like fire, crush, and shock.

\section{Goldsboro, North Carolina}

On January 24, 1961, during a B-52 airborne alert mission, structural failure of the right wing resulted in two weapons separating from the aircraft during aircraft breakup at 2,000 to 10,000 feet altitude. One bomb parachute deployed and the weapon received little impact damage. The other bomb fell free and broke apart upon impact. No explosion occurred.

\section{Palomares, Spain}

On January 17, 1966, a B-52 and a KC-135 collided during a routine high altitude air refueling operation. Both aircraft crashed near Palomares, Spain. The B-52 carried four nuclear weapons. One was recovered on the 


\section{DISCLAIMER}

This report was prepared as an account of work sponsored by an agency of the United States Government. Neither the United States Government nor any agency thereof, nor any of their employees, makes any warranty, express or implied, or assumes any legal liability or responsibility for the accuracy, completeness, or usefulness of any information, apparatus, product, or process disclosed, or represents that its use would not infringe privately owned rights. Reference herein to any specific commercial product, process, or service by trade name, trademark, manufacturer, or otherwise does not necessarily constitute or imply its endorsement, recommendation, or favoring by the United States Government or any agency thereof. The views and opinions of authors expressed herein do not necessarily state or reflect those of the United States Government or any agency thereof. 
ground; another was recovered from the sea on April 7 , after extensive search and recovery efforts. The other two of the weapons' high explosive materials exploded on impact with the ground, scattering some radioactive materials.

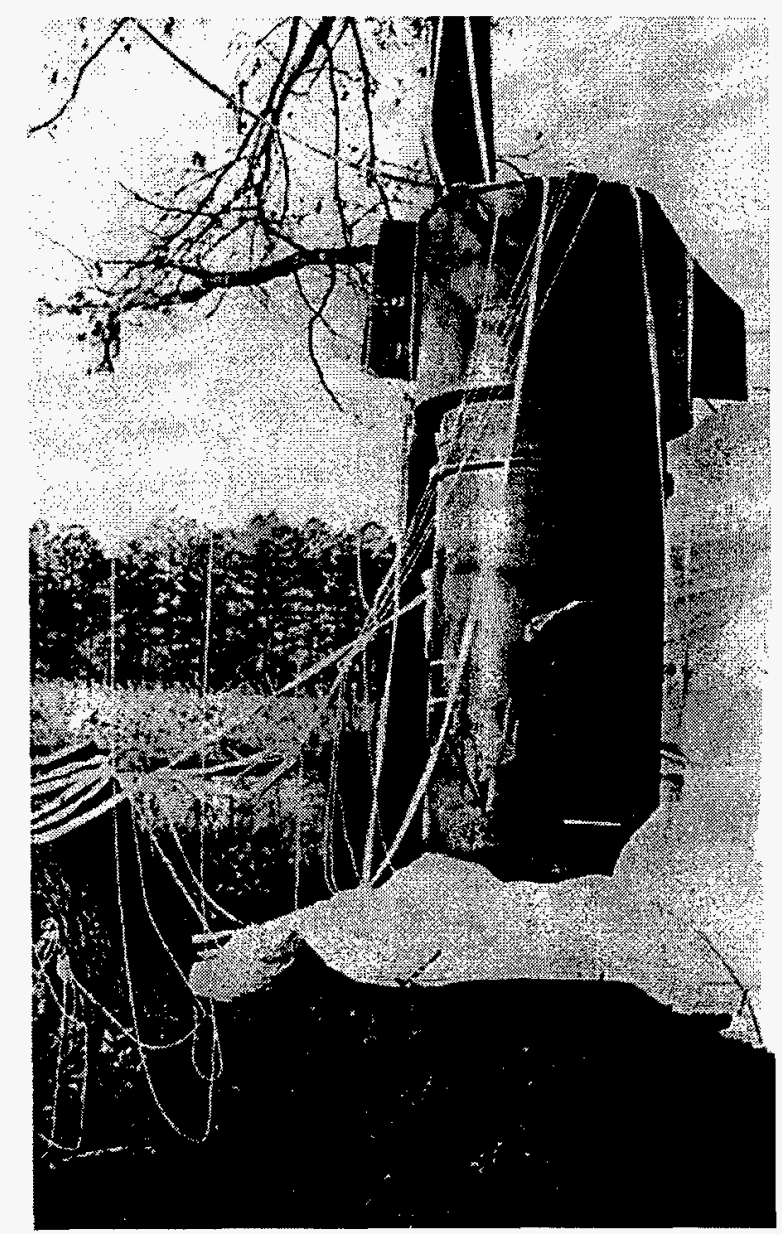

Figure 1. Accident at Goldsboro, NC

\section{Thule, Greenland}

On January 21, 1968, a B-52 from Plattsburgh AFB, New York, crashed and burned some seven miles southwest of the runway at Thule Airbase, Greenland, while approaching the base to land. The bomber carried four nuclear weapons, all of which were destroyed by fire.

\section{Damascus, Arkansas}

On September 19, 1980, during a routine maintenance in a Titan II silo, an Air Force repairman dropped a heavy wrench socket, which rolled off a work platform and fell toward the bottom of the silo. The socket bounced and struck the missile, causing a leak from a pressurized fuel tank. The missile complex and surrounding area were evacuated and a team of specialists was called in from Little Rock Air Force Base, the missile's main support base. About eight hours after the initial puncture, fuel vapors within the silo ignited and exploded. The missile burst through the silo door and landed about one thousand feet away. The missile's reentry vehicle, which contained a nuclear warhead, was recovered intact. There was no radioactive contamination.

\section{Early Weapon Safety Concepts}

In the 1950s, safety devices incorporated into weapons controlled electrical contacts. Firing signals were kept away from the critical components by an air gap. Most safety switches were operated by DC motors driven from $28 \mathrm{~V}$ aircraft power. Other devices derived their operational energy from the delivery environments, like missile acceleration. Electrical power was routed by cables and distributed by printed circuit boards. Engineers of the day addressed safety by using generous spacing between circuit paths and by isolating critical electrical cables from one another. ${ }^{2}$

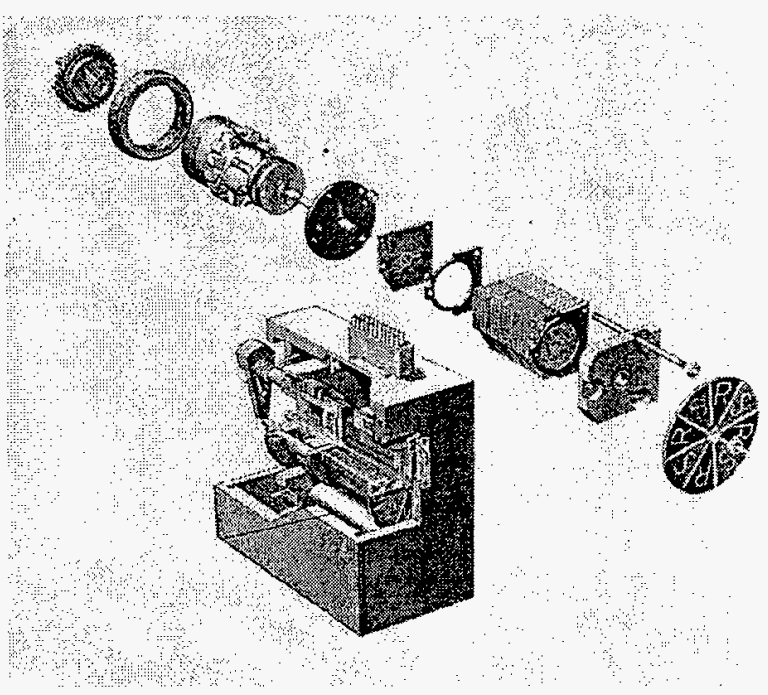

Figure 2. DC Motor Driven Safety Device

Safety was based on assumptions of how weapons would behave in abnormal environments. For example, engineers and analysts assumed that faults to ground would "dud" the system and that soldered joints would melt in a fire and create open circuits. This led to large probability matrices developed for environments based on the assumed response. Elaborate computer models and fault trees were generated to calculate the probability of an inadvertent detonation. Although the analyses became increasingly sophisticated, did they assure safety? Also, the models focused on the weapon response to a single 
environment. How could the effects of credible combinations of abnormal environments be addressed?

The methodology above suffered from a fatal flaw. There was no technical basis for some of the underlying assumptions. In fact, some were grossly misleading. For example, a fault to ground may not dud the system at all. It may simply create additional propagating damage leading to further unpredictability. The fundamental problem was expecting the accident to manufacture a "safety device or feature" from charred insulation or melted solder instead of deliberately engineering those responses.

Modern designs incorporate improved redundant safety features that are engineered to insure that a nuclear explosion does not occur as a result of an accident and that the weapon behave predictably in an unpredictable situation.

\section{The Principles of Enhanced Nuclear Detonation Safety (ENDS)}

The experiences of the 1950s and 1960s taught designers some important lessons. First, accidents frequently mimic the delivery mode of the weapon. How is a bomb to know if it was deliberately or inadvertently released from an airplane? Second, wire insulation and printed circuit boards become unpredictable during a fire and it becomes relatively "easy" to get $28 \mathrm{VDC}$ across the terminals of a safety device.

The identification of Enhanced Nuclear Detonation Safety principles evolved over an extended period of time. ${ }^{3}$ These principles reduce the number of components of the weapon system that are involved in nuclear safety. A salient feature of the concept is that a special region of the weapon system, which contains safety critical components, is designed to respond to abnormal environments in a predictably safe manner. Nuclear safety in the abnormal environment is achieved even though premature signals appear at the input of the special region and without a precise definition of the environments. The approaches permit verification of system safety by analysis and test at reasonable costs. The following characteristics, known as the " 3 Is," are integral parts of the weapon system concept:

The safety of nuclear weapons is based upon the principles of isolation, incompatibility, and inoperability. The critical components necessary for a nuclear detonation are to be isolated from their surroundings by placing them within an energy barrier. The deliberate action that passes through the barrier when a nuclear detonation is desired must be incompatible with naturally occurring signals, energy types, or levels. In accidents or in uncontrolled environments, before the energy barrier is breached, some of the components needed for nuclear detonation become inoperable.

Isolation

The critical components necessary for a nuclear detonation are isolated from their surroundings by placing them within a special region enclosed by an energy barrier. The volume enclosed by the barrier is known as the "exclusion region." The barrier blocks all forms of energy at the levels sufficient to cause a nuclear yield of greater than the equivalent of four pounds of TNT. A perfect barrier will make a weapon perfectly safe.

However, the result of perfect isolation is not a functional weapon. To initiate a nuclear detonation, some energy must be permitted inside the exclusion region. An energy control element is required. When the control element is closed, it forms an integral part of the barrier; when the control element is opened, it readily allows transfer of energy inside the exclusion region to cause a nuclear detonation. A nuclear weapon safety device must form a part of the exclusion region barrier and, on demand, provide an energy path into the exclusion region.

Incompatibility

Great care is taken to assure that only a deliberate action opens the energy control element. The action can originate from human intent or the delivery environments of the weapon. The nuclear weapon safety device serves as a combination lock that prevents weapon usage until the deliberate action occurs. The combination to the lock is a complex pattern of binary pulses. Each safety device contains one pattern and can only be operated by the application of that pattern. Typically, nuclear weapons contain two safety devices and each safety device is operated with a different pattern. These patterns are analyzed to ensure they are incompatible with naturally occurring signals and are known as unique signals. They are engineered so that the odds of their accidental generation from a naturally occurring source is far less than one chance in a million.

A nuclear weapon uses a mechanical device that forms a part of the exclusion region barrier and, upon receipt of the correct unique signal, provides a path to permit the transfer of energy into the exclusion region. The safety device is required to withstand assault from the environment and remain functional until specified critical environmental levels are surpassed. 
Inoperability

There are no perfect barriers. At some level of abnormal environmental exposure, the energy from the surroundings becomes so intense that the barrier loses integrity. That is, the barrier melts or ruptures. Nuclear safety is assured in this situation by incorporating environmental vulnerability into certain critical components. These components are known as weaklinks with the name derived from the truism, "A chain is only as strong as its weakest link." A weaklink must be functional for a nuclear detonation and is designed to fail at relatively low environmental levels. These levels are low enough to assure the weaklink fails before the safety device or barrier fails. Ideally, the weaklinks are collocated with the safety devices, so both components experience the same environmental assault. Capacitors, which store electrical energy used to fire weapon detonators, are common weaklinks. The capacitor is designed using materials that have a relatively low melting temperature. In a jet fuel fire, for example, the materials in the capacitor will melt and the capacitor will become irreversibly inoperable before the safety device or barrier fails from thermal exposure. In this way, a weapon fails in a safe condition and in a predictable fashion.

\section{Nuclear Weapon Safety Device Design Fundamentals}

Nuclear weapon safety devices are known as stronglinks since they are used in conjunction with weaklinks. The term stronglink is used since the devices are designed to be rugged and not readily circumvented in vibration, shock, fires, or electromagnetic fields. Stronglinks consist of four basic elements: a driver, a pattern discriminator, an energy control element, and a status monitor. ${ }^{4}$ Due to their predictable and deterministic nature, stronglinks have been and continue to be mechanical devices.

The desired motion within the stronglinks is created by solenoids or motors. The drivers can be actuated by simple DC or AC signals. For this reason, stronglinks separate the drivers from the energy control element with a pattern discrimination mechanism. The discriminator looks for motion from two independent drivers in a specified pattern. Each part of the motion is called an event and the pattern or code often consists of twenty-four events. The patterns are carefully analyzed so as not to be readily generated in expected or abnormal environments. Most stronglinks are single try devices. If the complex pattern is not received in the proper order, the mechanism will lock and additional inputs will neither advance it toward the closure of the energy control element nor restore it to the start of the signal pattern. The single try feature makes the proper operation of the stronglink incompatible with naturally occurring signals.

If the correct pattern is presented to the discriminator, it activates the energy control element and creates a low impedance pathway into the exclusion region. The energy control elements that have been used in stronglinks are electrical contacts, explosive pellets, ferrite buttons, and optical fibers or prisms.

In general, two stronglink mechanisms are arranged in series along the energy path. The mechanisms are of different types in order to gain independence and prevent common mode failures. It is desirable that not only the mechanism type be independent but also that the energy coupling and associated weaklinks be independent between the two stronglinks. This is not always done due to the difficulties imposed by volume limitations and acceptability of different weaklinks.

\section{Hardware Implementations of Stronglinks}

The first generation of stronglinks was developed about 25 years ago. The energy control elements were sets of electrical contacts. The two stronglinks are the MC2935 Trajectory Stronglink and MC2969 Intent Stronglink, shown in Figures 3 and 4 . When enabled, electrical energy would pass through the contacts and charge a capacitor in the firing system. The capacitor was identified as the thermal weaklink although the location was not always proximate and certain localized hot spots could compromise the stronglink before the capacitor failed irreversibly.

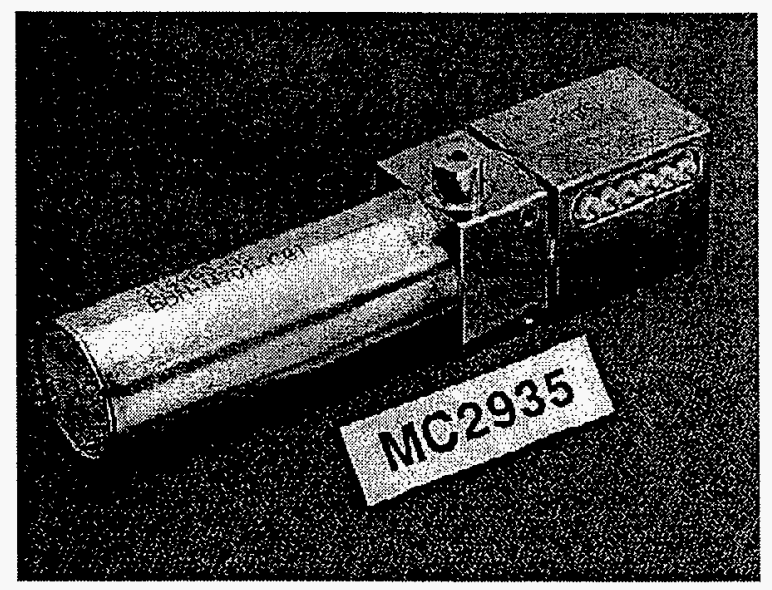

Figure 3. MC2935 Trajectory Stronglink 


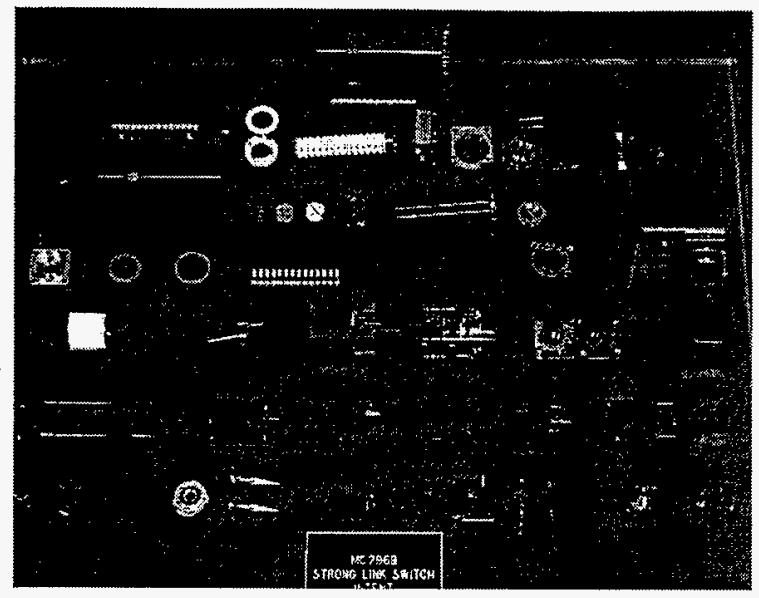

Figure 4. MC2969 Intent Stronglink.

The second generation of stronglinks was developed about 15 years ago. One motivation was to reduce the manufacturing cost and to increase the reliability by simplifying the mechanisms and reducing the number of parts. The second generation of stronglinks consisted of the detonator stronglink and dual magnetic stronglinks.

The major motivation for the development of the second generation of stronglinks was to improve the safety of the weapon. The detonator cables must exit the firing set and penetrate the barrier around the nuclear explosive package. The detonator cables must be protected in order not to bypass the stronglink function. Several possible accidents were proposed that exposed the detonator cables. For this reason, a detonator stronglink was developed by Lawrence Livermore National Laboratories. A small explosive pellet is rotated out of position between the slapper detonator and the main charge of high explosive. See Figure 5.

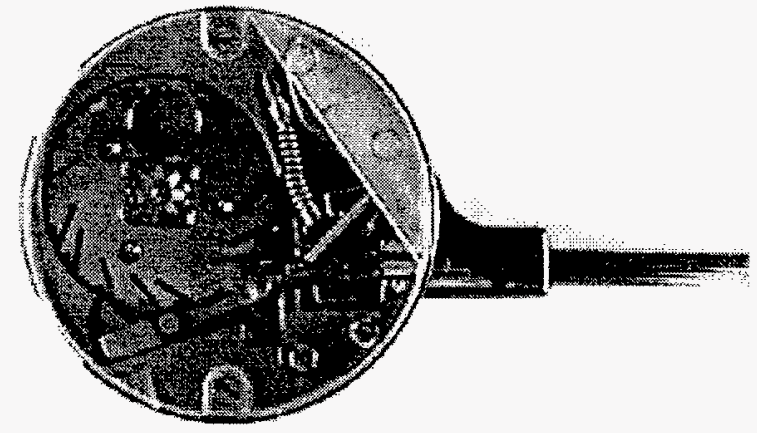

Figure 5. Detonator Pellet Stronglink Discriminator

The explosive pellet was the thermal weaklink. The actuator, not shown, used two linear solenoids that pulled or pushed on the mechanical cable attached to the discriminator.

Even in severe accidents that separate the firing set from the explosive package, one of the stronglinks keeps the detonator in a safe condition. The detonator stronglink was fielded in two weapons and was used in series with an electrical contact stronglink in the firing set.

A potential weakness of the electrical contact stronglinks is their susceptibility to high voltage breakdown. The dual magnetic stronglinks were developed to prevent any substantial energy from crossing the open magnetic switch under extreme environmental conditions. The two magnetic stronglinks were located in the middle of the firing set. Each stronglink contains half of a transformer and energy is controlled by moving highly permeable ferrite buttons between the transformer cores. The capacitor was the thermal weaklink. The dual magnetic stronglink was fielded in the last weapon to enter the nuclear weapon stockpile. See Figure 6.

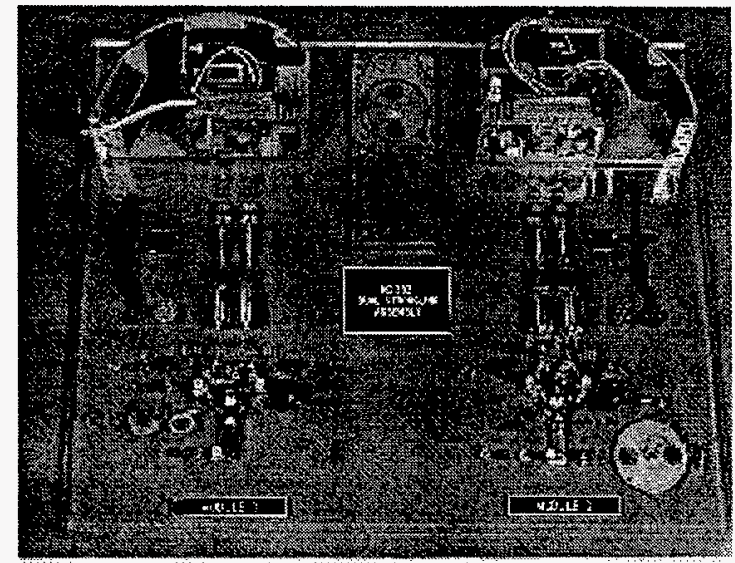

Figure 6. MC3831 Dual Stronglink

\section{$\underline{\text { Review of Nuclear Weapon Safety }}$}

As the armed services authorized new nuclear weapons to be built for specific military needs, there was a strong, prevailing motivation by the Department of Energy Oversight Committee to improve the safety of each successive weapon over the safety of the preceding weapon. Each new weapon, whether designed by Los Alamos National Laboratories or Lawrence Livermore National Laboratories, incorporated improved safety of the nuclear explosive package, as well as improvements in the arming, fuzing, firing, and safety systems provided by Sandia National Laboratories.

As the safety theme of the new weapon is developed and requirements for the components are issued, an 
independent assessment of the nuclear implementation is performed by an independent nuclear safety group, which is funded and managed independently of the system and component design groups. The safety evaluation does not rate the weapon as good or acceptable but outlines the departure from the nuclear safety principles. The nuclear safety evaluation critiques the system as well as components in the safety theme.

As the weapon continues in development and is ready for entry into the stockpile, a final safety evaluation is provided to the military and Department of Energy officials. Indeed, the primary reason for the national laboratories to be under control of the Department of Energy in the development of nuclear weapons is to give an equal footing of safety with wartime performance.

The Congress of the United States also authorizes independent safety reviews of the nuclear weapons. ${ }^{5}$ The multiple levels of nuclear weapon safety review allow safety weak points to be thoroughly considered by those not responsible for working the details or concerned about the wartime function of the weapon.

\section{The Cold War Ended - No New Weapons}

The ending of the cold war in the early 1990s caused the cancellation of all new weapons that were in fullscale development. Concepts for improved nuclear safety, such as direct optical initiation and paste extrudable explosive, continued in advanced development. As all underground nuclear testing in the U.S. is now prohibited, the physics labs (Los Alamos and Lawrence) have declined to certify any new weapons involving substantial changes to the nuclear explosive package. Advanced development ideas, such as a paste extrudable explosive that substantially altered the physics package, were subsequently dropped.

The nuclear weapon stockpile has been greatly reduced in accordance with international treaties, but there is an enduring stockpile that is maintained fully functional. Some of these weapons entered the stockpile about 30 years ago and are approaching their design life. The oldest weapons have no unique signal stronglinks, while the newer weapons have the second generation of unique signal stronglinks. Nuclear weapons have some components with lifetimes far shorter than the overall weapon lifetime. These short life components are regularly upgraded in overhauls. Some of the short life components have parts built with sunset technology and will be redesigned with modern technology. In addition, there is a strong effort in advanced development to design replacements for the safety systems of the older weapons that have no unique signal stronglinks. This is a technical challenge since the older systems have no space in the firing system for unique signal stronglinks or little space in the detonators for stronglinks. The arming, fuzing, and firing systems are being studied for size reduction in order to give space for stronglinks.

Direct optical initiation has continued in advanced development from 1991 and is being evaluated for firing and safety systems upgrades in the older weapons. Detonator stronglinks are receiving much attention to reduce their size and to incorporate the electromechanical drivers within the unique signal mechanism. See Figure 7. Piezoelectric motors are in development and current designs are $8-\mathrm{mm}$ diameter by $3-\mathrm{mm}$ thick. Low manufacturing variability and weapon environments are the chief focus of design teams. Highly customized rotary solenoids are also being considered for these small detonator stronglinks.

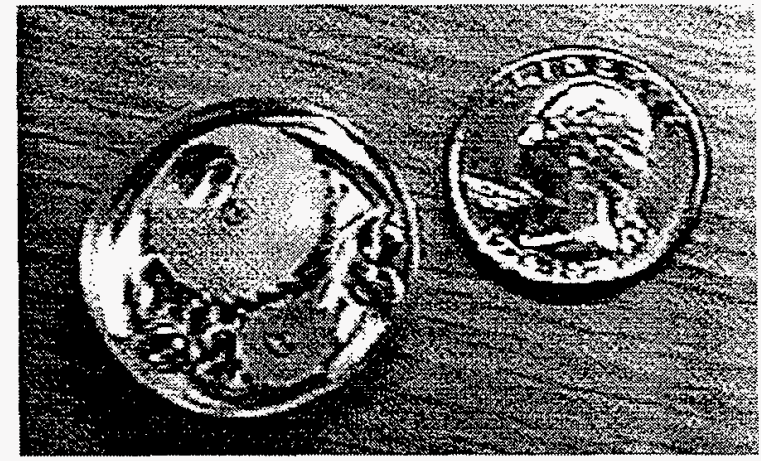

Figure 7. Advanced Development Detonator Stronglink

\section{Advanced Manufacturing Enables New Stronglinks}

New precision fabrication processes, most notably: multilevel polysilicon surface micromachining and LIGA (Lithographie, Galvanoformung, Abformung) hold promise for further reducing the volume required by a stronglink. Together, these processes extend the range of dimensions over which stronglinks can be designed and provide retrofit options not previously available. The key for each is the precision with which they realize features. It is exactly this precision that makes microscopic feature sizes possible.

\section{Multilevel Silicon Surface Micromachining}

Surface micromachining uses the planar fabrication techniques common to the microelectronic circuit fabrication industry to manufacture micromechanical devices. ${ }^{6}$ The standard building-block process consists of depositing and photolithographically patterning alternate 
layers of low-stress polycrystalline silicon and sacrificial silicon dioxide. At the completion of the process, the sacrificial layers, as their name suggests, are selectively etched away in hydrofluoric acid $(\mathrm{HF})$, which does not attack the polysilicon layers. The result is a construction system consisting of one layer of polysilicon which provides electrical interconnection and one or more independent layers of mechanical polysilicon that can be used to form mechanical elements ranging from simple cantilevered beams to complex systems of springs, linkages, mass elements and joints.

Surface Micromachining (SMM) produces machinery with dimensions in the $10-100$ s of micron range with feature sizes as small as 1 micron. SMM contains hundreds of processing steps. The greatest advantage of SMM is that the mechanisms are selfassembled and monolithically produced, thereby reducing cost and improving reliability. The chief disadvantages of SMM are: 1) the parts are highly planar, hundreds of microns in diameter but only a few micron thick, and 2) it is difficult to find suitable energy coupling elements for these micromechanisms. Optics is well suited for interfacing with micromachines. Sandia is developing an SMM stronglink based on the same discriminator used in the Detonator Surety Device (shown in Figure 7) with a pop-up mirror as an energy control element. See Figure 8.

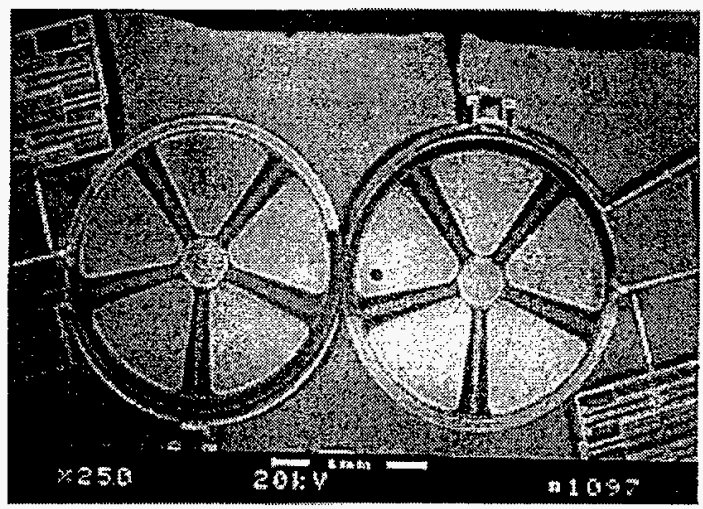

Figure 8. Polysilicon Surface Micromachined Countermeshing Gear Discriminator

$\underline{\text { LIGA }}$

LIGA (an acronym from German words for lithography, electroplating, and molding) is a micromachining technology originated in the early 1980s at the Karlsruhe Nuclear Research Center. ${ }^{7,8}$ Synchrotron Radiation is used to expose a thick (100 $1000 \mathrm{~mm}$ ) photoresist attached to a substrate. Polymethyl methacrylate (PMMA) is the typical photoresist material. The exposed PMMA is developed, leaving high-aspect ratio trenches. Metal can then be electroplated into the cavities in the PMMA. For many applications, the metal structures are released from the substrate to be used as freestanding parts. The metal structures can be used as molding dies into which plastic or powder materials are injected.

The next generation of stronglinks will be a hybrid of conventionally machined and LIGA parts. As the size of conventional stronglinks gets smaller, the tolerance associated with part profiles is becoming a large fraction of the nominal feature size. In electrical terms, nominal size-to-tolerance is equivalent to signal-to-noise. Traditionally, stronglink parts are made using material removal techniques that are available in most machine shops, such as end milling, precision screw machining, and wire-EDM (Electrical Discharge Machining). The practical feature size limit from conventional machining is around 200 microns (.008 inches). Tolerances on profiles are limited to 50 microns (.002 inches) and to 10 microns (.0004 inches) on holes. As machine parts get smaller, the signal-to-noise ratio approaches $4: 1$; backlash and other mechanical errors become increasingly important. LIGA is capable of reproducing features to within 2 microns. Using the same 4:1 ratio, a machine can have features less than 10 microns thereby allowing much smaller machinery. LIGA also comfortably produces structures with planar dimensions greater than 1 millimeter and employs a wide material base of metals and plastics. Since LIGA only produces prismatic parts, complex geometries must be assembled from multiple

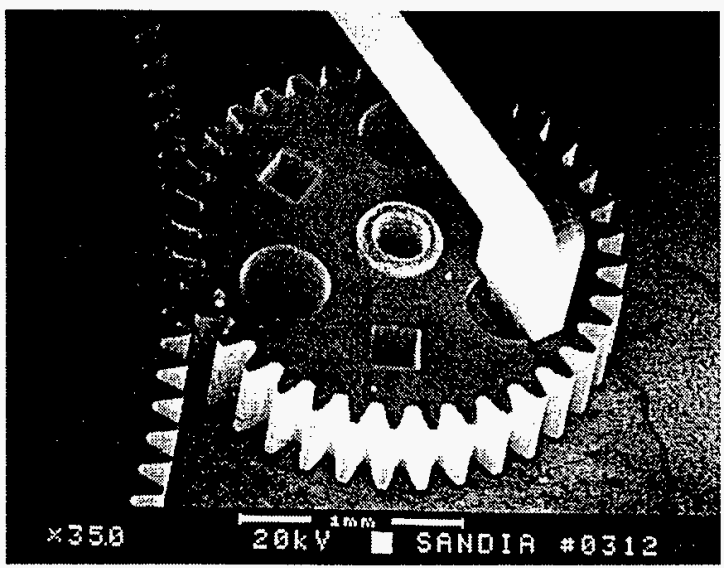

layers. See Figure 9.

Figure 9. Mechanical Gears fabricated with LIGA

$\underline{\text { Summary }}$ 
The extreme explosive potential of nuclear weapons resulted in a safety theme of separables in the early bombs. As weapons evolved from bombs to ballistic missiles and immediate launch capability, the safety theme turned to the idea of conventional weapons and protecting the detonator from firing signals. As experience with accidents increased, the safety theme of weaklinks and unique signal stronglinks was adopted to make the weapons more predictable in accidents. Current safety efforts are to make energy forms for detonation more specialized or to make bypass of the safety device more difficult.

Development of safety devices is focussed on size reduction to allow integration into existing systems.

\section{$\underline{\text { References }}$}

1. Narrative Summaries of Accidents Involving U. S. Nuclear Weapons, 1950 - 1980. Department of Defense. Accident Response Group Training Manual.

2. Nuclear Weapon System Safety Assessment, A Methodology Overview. NST 210 Course Notes. Sandia National Laboratories.

3. SC-DR-72-0492. Approaches for Achieving Nuclear Weapon Electrical System Safety in Abnormal Environments. Safety Assurance Studies Division. Sandia National Laboratories. August, 1972

4. SAND 93-0951. A Primer on Unique Signal Stronglinks. D. W. Plummer and W. H. Greenwood. Sandia National Laboratories. 1993.

5. S.D. Drell, J.S. Foster, and C. H. Townes (1990), Report of The Panel of Nuclear Weapons Safety, of the House Armed Services Committee.

6. Multi-Level Polysilicon SurfaceMicromachining Technology: Applications And Issues, J. J. Sniegowski, (Invited Paper) ASME 1996 International Mechanical Engineering Congress and Exposition, Proc. of the ASME Aerospace Division, November 17-22, 1996, Atlanta, GA, AD-Vol. 52, pp. 751-759.

7. E. W. Becker, W. Ehrfeld, P. Hagmann, A. Maner, and D. Munchmeyer. Microelectronic Eng. 4. 1986. pp. 35-56.
8. T. R. Christenson and H. Guckel. Micromachining and Microfabrication Process Technology. SPIE Proceedings, 2639. Austin, TX. 1995. pp. 134-145.

Sandia National Laboratories is a multiprogram laboratory operated by Sandia Corporation, a Lockheed Martin Company, for the United States Department of Energy under contract DE-AC04-94AL8500. 


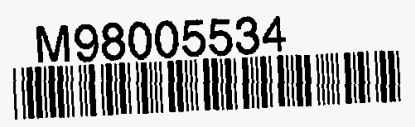

Report Number (14) SAND--98-1184C

Publ. Date (11)

Sponsor Code (18) DOE /MA, XF
UC Category (19) UC-900, DOE/ER 